\title{
A Duplex Real-Time RT-PCR System with an Internal Control Offers Sensitive and Reliable Broad-Spectrum Detection of Squash mosaic virus Variants
}

Rugang Li, United States Department of Agriculture-Agricultural Research Service (USDA-ARS), U.S. Vegetable Laboratory, Charleston, SC; Sven Berendsen, Rijk Zwaan Breeding B.V. De Lier, The Netherlands; and Kai-Shu Ling, USDA-ARS, U.S. Vegetable Laboratory, Charleston, SC

\begin{abstract}
Li, R., Berendsen, S., and Ling, K.-S. 2016. A duplex real-time RT-PCR system with an internal control offers sensitive and reliable broad-spectrum detection of Squash mosaic virus variants. Plant Dis. 100:625-629.

Squash mosaic virus (SqMV), a seedborne virus, belongs to the genus Comovirus in the subfamily Comovirinae of the family Secoviridae. SqMV has a bipartite single-stranded RNA genome (RNA1 and RNA2) encapsidated separately with two capsid proteins. With the recent identification of a third genotype in SqMV, a greater genetic diversity with only 88 to $89 \%$ sequence identity among them are recognized. With the existence of genetic diversity, a previously developed quantitative real-time reverse-transcription polymerase chain reaction (qRT-PCR) failed to detect isolates in this new genotype. Therefore, it was necessary to create a new qRT-PCR that would react with all SqMV isolates in three

different genotypes. From a multiple sequence alignment of the available SqMV sequences in GenBank, a conserved sequence segment in the $3^{\prime}$ untranslated region of RNA2 was identified for primer and probe design. A new qRT-PCR was developed, which provided broad-spectrum reactions to SqMV isolates, including those from the newly recognized third genotype. To improve the reliability in determining the sample quality and result interpretation, an internal amplification control with an endogenous gene sequence (18S ribosomal RNA) was successfully incorporated to develop a duplex qRT-PCR system that was useful for seed health test.
\end{abstract}

Squash mosaic virus (SqMV), in the genus Comovirus, subfamily Comovirinae of the family Secoviridae (Sanfaçco et al. 2012), is a well-recognized virus. With the increasing international seed trade activity, this seedborne virus may be introduced to a region or a field if the seed is not thoroughly tested. SqMV infection could result in a disease epidemic on cucurbit crops, including cucumber, melon, squash, pumpkin, and watermelon. Since its first discovery in California in 1934 (Kendrick 1934), SqMV has continually been reported in other U.S. states, including California (Alvarez and Campbell 1978; Grogan et al. 1959; Haudenshield and Palukaitis 1998; Ling et al. 2011; Milne et al. 1969), Arizona (Haudenshield and Palukaitis 1998; Nelson and Knuhtsen 1973a,b), Florida (Hiebert and Purcifull 1981), New York (Hu et al. 1993), and Illinois (Jossey and Babadoost 2008). Its occurrence has also been reported in a number of other countries, including Japan (Han et al. 2002; Yoshida et al. 1980), Australia (Coutts and Jones 2005), China (Hu et al. 2009), and Spain (Li et al. 2015).

SqMV genome consists of bipartite single-stranded RNA (RNA1 and RNA2) that are encapsidated separately with two capsid proteins. Both RNA molecules contain a genome-linked viral protein (VPg) and a poly (A) tail. VPg functions as a $5^{\prime}$ mRNA cap in translation initiation and, when uridylylated, as a primer for RNA synthesis by the virus-encoded RNA-dependent RNA polymerase (RdRp). RNA1 is translated into a single polyprotein that is processed into five functional proteins, including $32 \mathrm{~K}$ protease cofactor, $58 \mathrm{~K}$ helicase, $\mathrm{VPg}, 24 \mathrm{~K}$ protease, and $87 \mathrm{~K} \mathrm{RdRp}$. RNA2 is translated into two large overlapping polyproteins ( $52 \mathrm{~K}$ replication protein from the larger polyprotein and $42 \mathrm{~K}$ movement protein from the smaller polyprotein), followed by a larger coat protein and a smaller coat protein.

Studies on the genetic diversity of SqMV isolates initially divided them into two serotypes (Knuhtsen and Nelson 1968) and six biotypes

Corresponding author: K.-S. Ling; E-mail: Kai.Ling@ ARS.USDA.GOV

Accepted for publication 13 October 2015.

http://dx.doi.org/10.1094/PDIS-08-15-0944-RE

This article is in the public domain and not copyrightable. It may be freely reprinted with customary crediting of the source. The American Phytopathological Society, 2016.
(Nelson and Knuhtsen 1973b). However, a recent genome sequence analysis showed the presence of a greater genetic diversity ( $\mathrm{Li}$ et al. 2015). At least three genotypes, with only 88 to $89 \%$ nucleotide sequence identity among them, have been recognized (Haudenshield and Palukaitis 1998; Li et al. 2015). Presence of such broad genetic diversity and possible serological specificity among various genotypes poses a greater challenge to reliably detect SqMV isolates in seed and plant health tests. SqMV is a seedborne and seed-transmitted virus (Alvarez and Campbell 1978; Grogan et al. 1959; Milne et al. 1969). The virus is transmitted in the field to other cucurbit plants by beetles (Freitag 1956). In addition, its ease of mechanical transmission could result in a disease outbreak from cultivation activities. Seed transmission rates, ranging from $1 \%$ in commercial seed lots (Grogan et al. 1959; Milne et al. 1969) to an average of $10.6 \%$ in 62 experimental cantaloupe seed lots (Alvarez and Campbell 1978), have been reported. A typical seed health test for SqMV detection was conducted using enzymelinked immunosorbent assay (ELISA) (http://www.worldseed.org/cms/ medias/file/TradeIssues/PhytosanitaryMatters/SeedHealthTesting/ ISHI-Veg/Cucurbits_SqMV_CGMMV_MNSV_010811_2012.pdf). Due to the understanding of greater genetic diversity (Haudenshield and Palukaitis 1998; Li et al. 2015) and possible serological specificity (Knuhtsen and Nelson 1968) among SqMV isolates in different genotypes, a more sensitive and reliable molecular-based assay is urgently needed for seed health tests to meet the demand for increasing global seed trade activities on cucurbit seed.

To improve the efficiency in the seed health test, Ling and colleagues (2011) initially developed a TaqMan real-time quantitative reverse-transcription polymerase chain reaction (qRT-PCR, hereinafter designated as SqMV_2011) which was capable of detecting all SqMV isolates in both genotypes that were recognized at that time. Surprisingly, this qRT-PCR could not detect isolates in the recently recognized third genotype, RZ-SqMV ( $\mathrm{Li}$ et al. 2015). The complete genome sequence of this novel genotype was recently determined using the small RNA deep sequencing and assembly technology (Kreuze et al. 2009; Li et al. 2012, 2015). The RNA1 and RNA2 of RZ-SqMV had 5,858 and 3,370 nucleotides (nt), respectively. BLASTn sequence analysis of RZ-SqMV in the National Center for Biotechnology Information GenBank database showed only 87 to $88 \%$ nucleotide sequence identity in RNA1 and 84 to $86 \%$ in RNA2 to SqMV isolates in two other genotypes ( $\mathrm{Li}$ et al. 2015). 
Despite the presence of such strong sequence diversity among the three SqMV genotypes, several conserved sequence regions identified from a multisequence alignment could be suitable for primer and probe design. In this article, we report on the development of a new qRT-PCR (SqMV_2015) useful for a seed health assay to allow for a reliable and sensitive detection of diverse isolates of SqMV in all known genotypes.

\section{Materials and Methods}

Virus sources. In 2010, a new SqMV isolate (designated as RZSqMV), representing a third genotype, was collected from a squash field in Spain for evaluation of squash germplasm materials ( $\mathrm{Li}$ et al. 2015). Preliminary data showed that qRT-PCR (SqMV_2011 system), developed by Ling et al. (2011), even though it reacted to isolates in two known genotypes of SqMV at that time, failed to react to this new isolate. A virus culture of RZ-SqMV was obtained and established on melon plants in a greenhouse in Charleston, SC through mechanical inoculation. Another $14 \mathrm{SqMV}$-infected materials (leaf or seed samples) used for validation of the established qRT-PCR were supplied by collaborative seed companies, including nine seed samples from Peru, three leaf samples from Spain, and two samples from the Netherlands. A commercial melon seed lot ('Honeydew Green Flesh') from the United States, previously determined to contain an SqMV isolate (CA-SqMV) in genotype 1 (Ling et al. 2011), was also used for comparative analysis. Genotyping of those SqMV isolates in genotypes 1 and 2 was determined based on partial sequence information obtained from amplicons in two genomic regions using primers specific to the reference isolate M96148, targeting 440 to 968 or 1,619 to 2,077 nt, respectively. Although a majority of the tested isolates (11 of 14) were from genotype 1 , the isolate 13896.12 was a genotype 2 . Two other isolates (13896.11 and 13896.14) from Spain were previously determined to be genotype 3 ( $\mathrm{Li}$ et al. 2015).

RNA sample preparation. Total plant RNA was purified using a TRIzol reagent (Life Technologies). For RNA isolation from leaves, 0.2 to $0.5 \mathrm{~g}$ of leaf tissue in a Bioreba tissue extraction bag was processed in $1.5 \mathrm{ml}$ of TRIzol reagent using a Homex homogenizer (Bioreba). For RNA isolation from seed, $0.25 \mathrm{~g}$ of seed was first crushed with a hammer, then processed in $3 \mathrm{ml}$ of TRIzol reagent into slurry using Homex homogenizer. The resulting suspension was transferred into a 2-ml microtube and spun at $12,000 \times g$ for $10 \mathrm{~min}$. Approximately $1 \mathrm{ml}$ of supernatant was transferred into a new tube with $0.4 \mathrm{ml}$ of chloroform, which was shaken vigorously by hand for $15 \mathrm{~s}$ and left at room temperature for 2 to $15 \mathrm{~min}$. After centrifugation at $12,000 \times \mathrm{g}$ for $15 \mathrm{~min}$ at $4^{\circ} \mathrm{C}, 600 \mu \mathrm{l}$ of supernatant was transferred to a new tube, which was mixed with $1 \mathrm{ml}$ of isopropanol and left at room temperature for $10 \mathrm{~min}$. The mixture was centrifuged at $12,000 \times g$ for $10 \mathrm{~min}$ at $4{ }^{\circ} \mathrm{C}$. After pouring off the supernatant, the pellet was washed once with $70 \%$ ethanol, air dried for 5 to $10 \mathrm{~min}$, and redissolved in $200 \mu \mathrm{l}$ of RNase-free water. RNA concentration was measured using a NanoDrop spectrophotometer 2000 (Thermo Scientific) and serial dilutions were made with RNase-free water.

Primer and TaqMan probe design. To improve reliability in using qRT-PCR for sensitive detection of all genotypes of SqMV, a conserved sequence in the $3^{\prime}$ untranslated region (UTR; nucleotides 3,273 to 3,354 ) was identified from a multiple sequence alignment using six available RNA2 sequences, including RZ-SqMV. A new TaqMan probe (nucleotides 3,294 to 3,321: FAM-TCT TTCCCAGTTCGCTTTAGAAGCCTCT-TAMRA) and corresponding forward primer (nucleotides 3,275 to 3,293: $5^{\prime}$-TCTG GGTTTTGTTGTGGCT-3') and reverse primer (nucleotides 3,354 to 3,333: 5'-GCATCAAAGAAAACAAGCTCTT-3') were designed and synthesized by Integrated DNA Technologies (Fig. 1). In order to evaluate the quality of prepared RNA samples useful for real-time RT-PCR, an internal control with a plant endogenous sequence, $18 \mathrm{~S}$ ribosomal RNA (rRNA), was included in a duplex reaction. The 18S rRNA-specific TaqMan probe for internal control was labeled with a different fluorescent dye (CY5), with their sequences (449F: 5'-GTGACGGAGAATTAGGGTTCGA-3'; 498R: 5'-CTGCCTTCCTTGGATGTGGTA-3'; and 475_P: CY5-CCG GAGAGGGAGCCTGAGAAACGG-BHQ-2) derived from a previous publication (Osman et al. 2007). For comparative analysis, the old qRT-PCR (SqMV_2011 system) developed by Ling and colleagues (2011) was also included with its primers and TaqMan probe described in detail there.

Real-time RT-PCR. Each qRT-PCR assay was prepared using Takara's One-Step Ex Taq qRT-PCR kit in a reduced reaction volume of $10 \mu l$. Each simplex reaction consisted of $5 \mu l$ of $2 \times$ master reaction buffer, $0.25 \mu \mathrm{l}$ of Ex Taq HS mix ( $5 \mathrm{U} / \mu \mathrm{l}), 0.25 \mu \mathrm{l}$ of RTase $\operatorname{mix}(5 \mathrm{U} / \mu \mathrm{l}), 0.25 \mu \mathrm{l}$ of forward primer $(20 \mu \mathrm{M}), 0.25 \mu \mathrm{l}$ of reverse primer $(20 \mu \mathrm{M}), 0.25 \mu \mathrm{l}$ of TaqMan probe $(10 \mu \mathrm{M}), 0.19 \mu \mathrm{l}$ of ROX reference dye $(1 \times)$, and $0.5 \mu l$ of RNA preparation. For a duplex reaction, in addition to the above reaction components, $0.25 \mu \mathrm{l}$ of the forward primer $(20 \mu \mathrm{M}), 0.25 \mu \mathrm{l}$ of the reverse primer $(20 \mu \mathrm{M})$, and $0.25 \mu \mathrm{l}$ of the TaqMan probe $(10 \mu \mathrm{M})$ for the $18 \mathrm{~S}$ rRNA were also included in the reaction mixture. Real-time RT-PCR was carried out on a Stratagene Mx3000P detection system in a thermal cycling profile, with an initial RT cycle at $50^{\circ} \mathrm{C}$ for $30 \mathrm{~min}$ and denaturation at $95^{\circ} \mathrm{C}$ for $2 \mathrm{~min}$, then 40 cycles of $95^{\circ} \mathrm{C}$ for $10 \mathrm{~s}$ and $55^{\circ} \mathrm{C}$ for $30 \mathrm{~s}$. In the duplex reaction, both fluorescent signals (FAM and CY5) were measured simultaneously. In addition, for each experiment, both a nontemplate and a healthy sample were also used as negative controls.

Seed health assay. To determine the sensitivity and reliability of the new qRT-PCR (hereinafter referred to as SqMV_2015) for a seed health test, a commercial seed sample previously determined by ELISA to be infected by SqMV was used. With total RNA preparations extracted from 5, 10, and 100 seeds, along with two health control samples of 5 hybrid cantaloupe melon seeds and 2 pumpkin seeds, a duplex qRT-PCR, including an internal control 18S rRNA, was carried out accordingly. Each reaction was repeated at least three times.

\section{Results}

Comparative evaluation of two qRT-PCR systems. To determine relative sensitivity of the new qRT-PCR system (SqMV_2015), both RZ-SqMV (genotype 3) and CA-SqMV (genotype 1) were used as templates. Total plant RNA preparation in an RZ-SqMV-infected sample was adjusted to $100 \mathrm{ng} / \mu \mathrm{l}$ and then serially diluted with RNase-free water in 10-fold increments up to $1 \mathrm{pg} / \mu \mathrm{l}$ (Table 1 ).

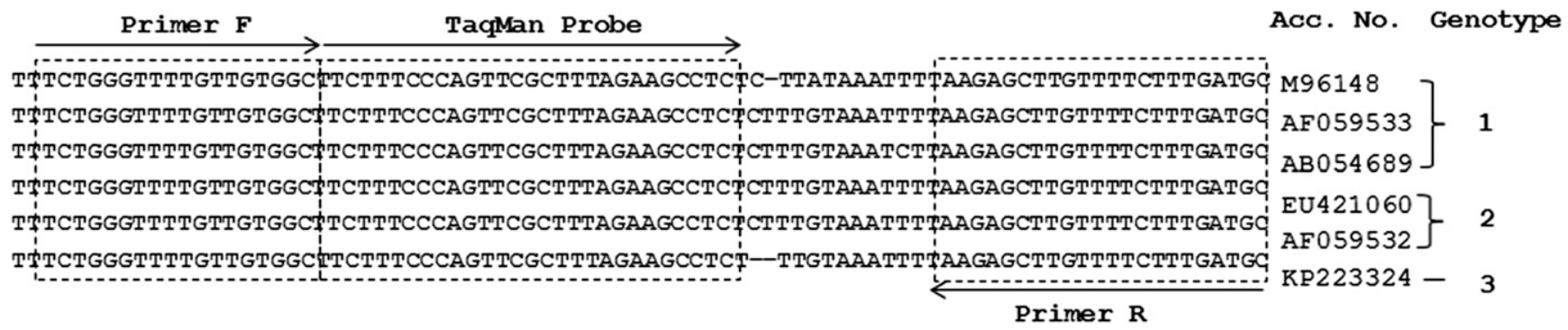

Fig. 1. Selection of a conserved sequence in the $3^{\prime}$ untranslated region to design TaqMan probe and primers in a new quantitative real-time reverse-transcription polymerase chain reaction (SqMV_2015) with broad-spectrum reaction to Squash mosaic virus (SqMV) isolates in all three genotypes. A multiple sequence alignment was generated to identify wellconserved sequences in the $3^{\prime}$ untranslated region of RNA-2 from six isolates belonging to genotype 1 (M96148, AB054689, and AF059533), genotype 2 (AF059532 and EU421060), and genotype 3 (KP223324). 
Table 1. Comparative sensitivity between the new quantitative reverse-transcription polymerase chain reaction (qRT-PCR) (SqMV_2015) and the old qRT-PCR (SqMV_2011) in detecting Squash mosaic virus (SqMV) isolates from two genotypes of SqMV ${ }^{a}$

\begin{tabular}{|c|c|c|c|c|c|c|}
\hline \multirow[b]{2}{*}{ Sample dilution ${ }^{\mathbf{b}}$} & \multicolumn{2}{|c|}{ Experiment 1} & \multicolumn{2}{|c|}{ Experiment 2} & \multicolumn{2}{|c|}{ Experiment 3} \\
\hline & SqMV_2015 & SqMV_2011 & SqMV_2015 & SqMV_2011 & SqMV_2015 & SqMV_2011 \\
\hline \multicolumn{7}{|c|}{ RZ-SqMV RNA (genotype 3) } \\
\hline $1: 100$ & 21.83 & $\mathrm{No} C t$ & 22.49 & $\mathrm{No} C t$ & 22.04 & $\mathrm{No} C t$ \\
\hline $1: 1,000$ & 25.80 & $\mathrm{No} C t$ & 24.92 & $\mathrm{No} C t$ & 25.52 & $\mathrm{No} C t$ \\
\hline $1: 10,000$ & 29.78 & No $\mathrm{Ct}$ & 28.59 & No $\mathrm{Ct}$ & 29.21 & No $\mathrm{Ct}$ \\
\hline $1: 100,000$ & 33.74 & No Ct & 32.72 & No Ct & 32.43 & No Ct \\
\hline $1: 1,000,000$ & 37.20 & No Ct & 36.60 & No Ct & 35.65 & No Ct \\
\hline $1: 10,000,000$ & No Ct & No Ct & No Ct & No Ct & No Ct & No Ct \\
\hline \multicolumn{7}{|c|}{ CA-SqMV RNA (genotype 1) } \\
\hline $1: 1$ & 11.94 & 15.21 & 13.53 & 15.80 & 13.86 & 15.56 \\
\hline $1: 10$ & 16.28 & 17.87 & 15.69 & 18.21 & 16.04 & 18.60 \\
\hline $1: 100$ & 19.12 & 21.43 & 18.71 & 20.83 & 19.02 & 21.53 \\
\hline $1: 1,000$ & 22.72 & 24.80 & 22.81 & 24.55 & 22.73 & 24.57 \\
\hline $1: 10,000$ & 28.60 & 29.99 & 32.32 & 33.22 & 33.92 & 35.57 \\
\hline $1: 100,000$ & 32.38 & 35.51 & 34.94 & 36.88 & $\mathrm{No} C t$ & No Ct \\
\hline $1: 1,000,000$ & 36.79 & 37.87 & $\mathrm{No} C t$ & $\mathrm{No} C t$ & $\mathrm{No} C t$ & $\mathrm{No} C t$ \\
\hline $1: 10,000,000$ & $\mathrm{No} C t$ & $\mathrm{No} C t$ & $\mathrm{No} C t$ & $\mathrm{No} C t$ & $\mathrm{No} C t$ & $\mathrm{No} C t$ \\
\hline $1: 100,000,000$ & No $\mathrm{Ct}$ & $\mathrm{No} C t$ & $\mathrm{No} C t$ & $\mathrm{No} C t$ & $\mathrm{No} C t$ & $\mathrm{No} C t$ \\
\hline Nontemplate $\left(\mathrm{H}_{2} \mathrm{O}\right)$ & No Ct & No Ct & $\mathrm{No} C t$ & No Ct & No Ct & No Ct \\
\hline
\end{tabular}

a $\mathrm{Ct}=$ cycle threshold.

b Dilution 1:100 was equivalent to the purified plant RNA preparation at $100 \mathrm{ng} / \mu \mathrm{l}$.

Table 2. Development of a sensitive and reliable duplex quantitative reverse-transcription polymerase chain reaction system (SqMV_2015) for a seed health assay to detect Squash mosaic virus (SqMV), with the $18 \mathrm{~S}$ ribosomal RNA (rRNA) as an internal controla

\begin{tabular}{|c|c|c|c|c|c|c|c|c|}
\hline \multirow[b]{2}{*}{ Preparations $^{\mathbf{b}}$} & \multicolumn{2}{|c|}{ Experiment $1(\mathrm{Ct})$} & \multicolumn{2}{|c|}{ Experiment $2(\mathrm{Ct})$} & \multicolumn{2}{|c|}{ Experiment $3(\mathrm{Ct})$} & \multicolumn{2}{|c|}{ Means Ct } \\
\hline & $\overline{\text { CY5/18S rRNA }}$ & $\overline{\text { FAM/SqMV }}$ & $\overline{C Y 5 / 18 S \text { rRNA }}$ & $\overline{\text { FAM/SqMV }}$ & $\overline{C Y 5 / 18 S \text { rRNA }}$ & $\overline{\text { FAM/SqMV }}$ & $\overline{C Y 5 / 18 S ~ r R N A ~}$ & $\overline{\text { FAM/SqMV }}$ \\
\hline 5 seeds & 10.85 & 13.92 & 11.12 & 13.44 & 11.60 & 14.56 & 11.19 & 13.97 \\
\hline 10 seeds & 10.43 & 11.99 & 11.27 & 12.89 & 11.13 & 13.47 & 10.94 & 12.78 \\
\hline 100 seeds & 11.61 & 16.54 & 11.76 & 15.24 & 11.87 & 16.08 & 11.75 & 15.95 \\
\hline CK1 & 10.12 & No Ct & 10.02 & No $\mathrm{Ct}$ & 9.77 & No Ct & 9.97 & No $\mathrm{Ct}$ \\
\hline $\mathrm{CK} 2$ & 11.04 & No Ct & 11.39 & No Ct & 10.74 & No $\mathrm{Ct}$ & 11.05 & No Ct \\
\hline $\mathrm{CK}+$ & 17.47 & 21.62 & 17.26 & 20.49 & 17.40 & 20.74 & 17.38 & 20.95 \\
\hline
\end{tabular}

${ }^{\mathrm{a}} \mathrm{Ct}=$ cycle threshold.

${ }^{\mathrm{b}} \mathrm{CK} 1=$ RNA preparations prepared from healthy hybrid cantaloupe melon seed, $\mathrm{CK} 2=$ RNA preparations prepared from healthy pumpkin seed, and CK $+=$ RNA preparation prepared from RZ-SqMV (genotype 3)-infected melon tissue. RNA template concentration was $100 \mathrm{ng} / \mu \mathrm{l}$ for all samples.

Table 3. Comparative analysis between the new quantitative reverse-transcription polymerase chain reaction (qRT-PCR) (SqMV_2015) and the old qRT-PCR (SqMV_2011) for detection of Squash mosaic virus (SqMV)-infected seed and tissue samples

\begin{tabular}{|c|c|c|c|c|c|c|c|}
\hline Isolate designation & Genotype $^{\mathbf{a}}$ & Country of origin & Crop species & Tissue type & Year of collection & $\begin{array}{c}\text { qRT-PCR } \\
\text { (SqMV_2015) }\end{array}$ & $\begin{array}{c}\text { qRT-PCR } \\
\text { (SqMV_2011) }\end{array}$ \\
\hline 13896.1 & 1 & Peru & Melon & Seed & 2011 & 19.72 & 21.26 \\
\hline 13896.2 & 1 & Peru & Melon & Seed & 2011 & 18.53 & 18.89 \\
\hline 13896.3 & 1 & Peru & Melon & Seed & 2011 & 25.37 & 27.50 \\
\hline 13896.4 & 1 & Peru & Melon & Seed & 2011 & 26.24 & 28.28 \\
\hline 13896.5 & 1 & Peru & Melon & Seed & 2011 & 23.88 & 24.57 \\
\hline 13896.6 & 1 & Peru & Melon & Seed & 2011 & 24.89 & 25.15 \\
\hline 13896.7 & 1 & Peru & Melon & Seed & 2010 & 16.34 & 19.70 \\
\hline 13896.8 & 1 & Peru & Melon & Seed & 2010 & 18.90 & 22.00 \\
\hline 13896.9 & 1 & Netherlands & Rootstock & Seed & 2012 & 17.04 & 17.60 \\
\hline 13896.10 & 1 & Netherlands & Rootstock & Leaf & 2012 & 14.63 & 15.62 \\
\hline 13896.11 & 3 & Spain & Melon & Leaf & 2010 & 13.02 & No $\mathrm{Ct}$ \\
\hline 13896.12 & 2 & Spain & Melon & Leaf & 2012 & 13.86 & 14.77 \\
\hline 13896.13 & 1 & Peru & Melon & Seed & 2010 & 12.96 & 15.35 \\
\hline 13896.14 & 3 & Spain & Squash & Leaf & 2010 & 22.83 & No $\mathrm{Ct}$ \\
\hline Nontemplate & & & - & - & - & No Ct & No Ct \\
\hline
\end{tabular}

${ }^{\text {a }}$ Genotype of these isolates was determined based on partial sequence information obtained on PCR products amplified in two sequence regions using primers specific to M96148 targeting 440 to 968 or 1,619 to 2,077 nucleotides. Phylogenetic relationship analysis was carried out using the unweighted pair-group method with arithmetic means, Jukes-Cantor nucleotide distance measure, and a bootstrap of 100 replicates. Representative isolates for each genotype-genotype 1 (GenBank accession numbers M96148, AB054689, and AF059533), genotype 2 (AF059532 and EU421060), and genotype 3 (KP223324)—were used for alignments to determine their respective genotypes for the test isolates. 
On the other hand, the CA-SqMV was a cDNA product generated from $320 \mathrm{ng}$ of total RNA in a $20-\mu \mathrm{l}$ reaction volume. This cDNA product was diluted in 10 -fold increments with RNase-free water up to $1 \times 10^{-8}$ (Table 1). An aliquot of $0.5 \mu$ l of each dilution was added to each qRT-PCR. These comparative experiments were repeated three times (Table 1). The results showed that the SqMV_2015 qRT-PCR system was able to detect both genotypes at $1 \times 10^{-4}$ to $1 \times 10^{-6}$ dilutions (Table 1 ). However, the old SqMV_2011 qRT-PCR system (Ling et al. 2011) could only detect genotype 1 (CA-SqMV) isolates but not genotype 3 (RZ-SqMV) isolates (Table 1). Remarkably, the SqMV_2015 qRT-PCR system generated stronger fluorescent signals with approximately 1 to 3 cycle threshold $(\mathrm{Ct})$ values less (more sensitive) than that of the SqMV2011 qRT-PCR system using the same preparation for detection of SqMV in CA-SqMV preparations (Table 1).

Development of a duplex qRT-PCR system with an internal $18 \mathrm{~S}$ rRNA control to improve sample integrity and detection reliability. RNA integrity is a pivotal factor for detection reliability. Poor quality of RNA preparation often resulted in high $\mathrm{Ct}$ value and even no $\mathrm{Ct}$ value. To enhance the detection reliability, $18 \mathrm{~S}$ rRNA-specific probe and primers were included in each reaction. One preparation from five healthy hybrid cantaloupe melon seeds and another from two healthy pumpkin seeds were also included as negative controls. Consistent strong $\mathrm{Ct}$ values (10.94 to 11.75) generated in the duplex qRT-PCR system (Table 2) from 18S rRNA internal controls in healthy samples and in virus-infected seed preparations $(5,10$, and 100 seeds) suggested that the quality of RNA preparations was suitable for qRT-PCR. Thus, such low $\mathrm{Ct}$ values obtained from those test samples indicated that those seed samples did, indeed, carry SqMV. Therefore, those other samples with nondetectable levels of readings (no $\mathrm{Ct}$ ) could be unambiguously called SqMV free (Table 2).

Application of the SqMV_2015 qRT-PCR for seed and plant health tests. In this study, the new TaqMan RT-PCR (SqMV_2015) developed was capable of detecting SqMV isolates in diverse genotypes. Application of this sensitive and reliable SqMV detection system should help the seed industry to make a science-based decision on an SqMV-contaminated seed lot or an infected crop. Therefore, any potential introduction of a contaminated seed lot due to a likely poor result from possible lower sensitivity using ELISA could be minimized using this highly sensitive and reliable virus detection technology. The duplex detection system with an internal control should also offer additional advantages to evaluate the quality of RNA preparation as well as the reliability of the test results.

To determine whether the newly developed SqMV_2015 qRTPCR system could offer a broad-spectrum reaction to various SqMV isolates collected around the world, seed and leaf samples from Peru, Spain, and the Netherlands that represent three different SqMV genotypes based on partial sequencing were included for testing. Results showed that the SqMV_2015 qRT-PCR system was capable of detecting all SqMV isolates in three genotypes (Table 3). On the other hand, the SqMV_2011 qRT-PCR system was not able to detect two SqMV samples (13896.11 and 13896.14) belonging to genotype 3 (Table 3). Confirmation of these isolates as genotype 3 of SqMV was determined using genome sequencing, as previously reported (Li et al. 2015).

\section{Discussion}

In the present study, a sensitive and broad-spectrum qRT-PCR system (SqMV_2015) was developed to allow for a sensitive detection of all genotypes of SqMV, including the newly recognized genotype 3 (Li et al. 2015). Such improvement in SqMV detection was necessary due to the understanding of a possible serological specificity (Knuhtsen and Nelson 1968) as well as the recognition of a greater genetic diversity ( $\mathrm{Li}$ et al. 2015). With the existence of greater genetic diversity of SqMV, the previously developed qRTPCR system (SqMV_2011) that recognized isolates from genotypes 1 and 2 (Ling et al. 2011) would need improvement to allow for detection of all isolates, including those from genotype 3 . In the development of this new SqMV_2015 qRT-PCR detection system, a very well-conserved sequence region identified in the $3^{\prime}$ UTR of RNA2 was used. On the other hand, the old SqMV_2011
qRT-PCR detection system was developed based on a relatively conserved sequence region selected in the coding region of RNA2 for a small coat protein (Ling et al. 2011). Apparently, sequences in the $3^{\prime}$ UTR were more conserved among various isolates of SqMV in different genotypes than those in the coding region. This may explain why the newly developed qRT-PCR (SqMV_2015) was more sensitive and reliable in detecting diverse isolates of SqMV. In addition, by incorporating an internal amplification control of 18S rRNA (Osman et al. 2007), the duplex qRT-PCR system could be reliably used in seed and plant health assays. Such a duplex system not only would allow assessment of the sample RNA preparation quality but also an accurate interpretation of the results.

Understanding and characterizing the genetic diversity of a virus is important in developing a sensitive and reliable PCR-based detection method. For SqMV, the SqMV_2011 qRT-PCR system was developed based on two known genotypes at that time and was capable of detecting both genotypes in seed and plant health tests (Ling et al. 2011). With recent identification and sequencing of RZ-SqMV, a third genotype was recognized (Li et al. 2015). The newly developed SqMV_2015 qRT-PCR system was created based on the current knowledge of the SqMV genetic diversity in three genotypes. As more SqMV isolates from the world are collected and sequenced, it is possible that other genotypes could be identified. If that is the case, then additional improvements to qRT-PCR might be necessary to detect those emerging isolates with greater confidence.

A reliable disease diagnosis is dependent on accurate and sensitive virus detection. With technology improvement and more affordable real-time PCR equipment and reagents, qRT-PCR has been broadly adapted for plant virus detection. With the existence of genetic diversity, selection of conserved sequence regions that could be suitable for primer and probe design is important. Improvement to the qRT-PCR detection technology should be based on the wellcharacterized virus genetic diversity and validation of the technology on field-collected samples. In addition, the design strategy in using a duplex qRT-PCR with an internal amplification control may have general implications for improving the reliability of qRT-PCR on virus detection in routine disease diagnosis.

\section{Acknowledgments}

We thank A. Gilliard for technical assistance. This work was supported, in part, by USDA NIFA-SCRI (SCRI 2010-600-25320 and 2012-01507-229756) to K.-S. Ling.

\section{Literature Cited}

Alvarez, M., and Campbell, R. N. 1978. Transmission and distribution of squash mosaic virus in seeds of cantaloupe. Phytopathology 68:257-263.

Coutts, B. A., and Jones, R. A. C. 2005. Incidence and distribution of viruses infecting cucurbit crops in the Northern Territory and Western Australia. Aust. J. Agric. Res. 56:847-858.

Freitag, J. H. 1956. Beetle transmission, host range, and properties of squash mosaic virus. Phytopathology 46:73-81.

Grogan, R. G., Hall, D. H., and Kimble, K. A. 1959. Cucurbit mosaic viruses in California. Phytopathology 49:366-376.

Han, S. S., Yoshida, K., Karasev, A. V., and Iwanami, T. 2002. Nucleotide sequence of a Japanese isolate of Squash mosaic virus. Arch. Virol. 147:437-443.

Haudenshield, J. S., and Palukaitis, P. 1998. Diversity among isolates of squash mosaic virus. J. Gen. Virol. 79:2331-2341.

Hiebert, E., and Purcifull, D. E. 1981. Mapping of the two coat protein genes on the middle RNA component of squash mosaic virus (comovirus group). Virology 113:630-636.

Hu, J., Zhou, T., Liu, L., Peng, B., Li, H., Fan, Z., and Gu, Q. 2009. The genomic sequences of a Chinese isolate of Squash mosaic virus with novel $5^{\prime}$ conserved ends. Virus Genes 38:475-477.

Hu, J. S., Pang, S. Z., Nagpala, P. G., Siemieniak, D. R., Slightom, J. L., and Gonsalves, D. 1993. The coat protein genes of squash mosaic virus: Cloning, sequence analysis, and expression in tobacco protoplasts. Arch. Virol. 130: $17-31$.

Jossey, S., and Babadoost, M. 2008. Occurrence and distribution of pumpkin and squash viruses in Illinois. Plant Dis. 92:61-68.

Kendrick, J. B. 1934. Cucurbit mosaic transmitted by muskmelon seed. Phytopathology 24:820-823.

Knuhtsen, H. K., and Nelson, M. R. 1968. Identification of two serotypes in squash mosaic virus strains. Phytopathology 58:345-347. 
Kreuze, J. F., Perez, A., Untiveros, M., Quispe, D., Fuentes, S., Baker, I., and Simon, R. 2009. Complete viral genome sequence and discovery of novel viruses by deep sequencing of small RNAs: A generic method for diagnosis, discovery and sequencing of viruses. Virology 388:1-7.

Li, R., Gao, S., Berendsen, S., Fei, Z., and Ling, K.-S. 2015. Complete genome sequence of a novel genotype of Squash mosaic virus infecting squash in Spain. Genome Announc. 3:e01583-14.

Li, R., Gao, S., Hernandez, A. G., Wechter, W. P., Fei, Z., and Ling, K.-S. 2012. Deep sequencing of small RNAs in tomato for virus and viroid identification and strain differentiation. PLoS One 7:e37127.

Ling, K.-S., Wechter, W. P., Walcott, R., and Keinath, A. 2011. Development of a real-time RT-PCR assay for Squash mosaic virus useful for broad spectrum detection of various serotypes and its incorporation in a multiplex seed health assay. J. Phytopathol. 159:649-656.

Milne, K. S., Grogan, R. G., and Kimble, K. A. 1969. Identification of viruses infecting cucurbits in California. Phytopathology 59:819-828.
Nelson, M. R., and Knuhtsen, H. K. 1973a. Squash mosaic virus variability: Epidemiological consequences of differences in seed transmission frequency between strains. Phytopathology 63:918-920.

Nelson, M. R., and Knuhtsen, H. K. 1973b. Squash mosaic virus variability: Review and serological comparisons of six biotypes. Phytopathology 63:920-926.

Osman, F., Leutenegger, C., Golino, D., and Rowhani, A. 2007. Real-time RTPCR (TaqMan ${ }^{\circledR}$ ) assays for the detection of Grapevine leafroll associated viruses 1-5 and 9. J. Virol. Methods 141:22-29.

Sanfaçco, H., Iwanami, T., Karasev, A. V., van der Vlugt, R., Wellink, J., Wetzel, T., and Yoshikawa, N. 2012. Secoviridae. Pages 881-899 in: Virus Taxonomy: Ninth Report of the International Committee on Taxonomy of Viruses. A. M. O. King, E. Lefkowitz, M. J. Adams, and E. B. Carstens, eds. Elsevier/Academic Press, London.

Yoshida, K., Goto, T., Nemoto, M., and Tsuchizaki, T. 1980. Squash mosaic virus isolated from melon (Cucumis melo L.) in Hokkaido. Ann. Phytopathol. Soc. Jpn. 46:349-356. 